\title{
A novel approach to approximate fractional derivative with uncertain conditions
}

\begin{abstract}
This paper focuses on providing a new scheme to find the fuzzy approximate solution of fractional differential equations (FDEs) under uncertainty. The Caputo-type derivative base on the generalized Hukuhara differentiability is approximated by a linearization formula to reduce the corresponding uncertain FDE to an ODE under fuzzy concept. This new approach may positively affect on the computational cost and easily apply for the other types of uncertain fractional-order differential equation. The performed numerical simulations verify the proficiency of the presented scheme.
\end{abstract}

Keyword: Fractional differential equations; Caputo-type derivative; Laplace transforms; Basset problem; Uncertainty 